\title{
On the effects of the reactive terms in the Boltzmann equation
}

\author{
C. J. Zamlutti \\ Instituto Nacional de Psequisas Espaciais-INPE, Av. dos Astronautas, 1758, C.P. 515, 12201-970-São José dos Campos, SP, Brazil
}

Received: 17 August 1994/Revised: 13 June 1996/Accepted: 3 July 1996

\begin{abstract}
The effects of the production and loss mechanisms that affect the Boltzmann equations are considered by the inclusion of a reactive term. The necessary elements to develop a proper form for this term are revised and the curent trends analyzed. Although no accurate theoretical treatment of the problem is possible due to the many body nature of it, important relations can be derived which, besides being representative of the quantitative aspects of the matter, are illustrative of the qualitative features of the phenomenon. The overall procedure is detailed in this revision.
\end{abstract}

\section{Introduction}

The solar energy (solar radiation or energetic solar particles) acting on the terrestrial upper atmosphere produces chemical reactions of its constituent gases. As a result particles of a given species $s$ are produced by a set of chemical reactions and lost by other reactions. This is accounted for by the inclusion of a production-loss disturbing term in the right hand side (RHS) of the Boltzmann equation (see revision in Zamlutti, 1994). The development of a proper form for this term is still a matter of open discussion (see Eccles and Raitt, 1992).

The earliest account of the inclusion of the effects of production and loss mechanisms in upper atmosphere modeling was the heuristical inclusion of a heating term on the RHS of the energy equation (e.g., Bates, 1956). While satisfactory from a macroscopic perspective this term could not usually be obtained with confidence from microscopic laws (see revision in Torr et al., 1981). Burgers $(1964,1969)$ set down the foundations for a kinetic reactive collision term from which transport collision terms

$\overline{\text { Correspondence }}$ to: C. J. Zamlutti could be derived. A brief review on the present situation was presented by Eccles and Raitt (1992).

The importance of the present matter stem from the fact that the energy absorbed by the upper atmosphere particles is the driving source of all the dynamical and chemical phenomena of our environment. Unfortunately to date no complete work has been published that could give the reader a fair idea of the full extent of the problem. We will try to remedy this situation with this work.

The first problem one faces to model a phenomenon is the choice of a prospective mathematical expression to describe the causality relation between a known action and its produced result. Regarding this aspect the collisional form of Burgers (1969) improved by Eccles and Raitt (1992) has proven to be both adequate for modeling many reactive environments (Eccles and Raitt, 1992) and convenient for use (Zamlutti, 1994) to represent the production and loss of upper atmosphere particles species. However, it has been derived for particleparticle interactions and extended to include radiationparticle problems without detailing the whole extent of this matter.

In this revision we attempted to present this complex matter with a twofold purpose formalism of being at the same time more rigorous and comprehensive. To do this we had to resort to semiclassical mechanics to justify the use of our classical mechanics approach for a quantum mechanics problem. Next we started extending the concepts of binary elastic collisions to derive our model. The BGK collisional form of Burgers $(1964,1969)$ appears then as the balance between production and loss of a given particle. The material organization follows the same order. In Sect. 2 a brief survey on the elements of semiclassical mechanics necessary for the work, is included. Section 3 shows the basic mathematical formalism of the model. In Sect. 4 we comment on the theoretical problems of our approach. Section 5 contains a brief discussion on how our theory compares with traditional and new approaches of the current literature. 


\section{A brief survey of semiclassical mechanics}

Semiclassical mechanics is a method of solving problems of quantum mechanics using approximated solutions obtained with the techniques of classical mechanics. It is extensively used in problems of atomic and molecular collisions to reduce the involved complexity. It is grounded on the equivalence of the equations. Its elements will be introduced with particular application to our problem of upper atmosphere particle transformations.

We will consider generically any alteration in which a particle undergoes a change in its internal structure at an encounter such that an incident element, $a$, acting on a target particle, $b$, modifies the internal structure of this producing a transformed particle, $b^{\prime}$, as well as eventually other products, $c^{\prime}$, and itself undergoing a change to $a^{\prime}$. Primed symbols denote after encounter characteristics. This process is symbolically represented as:

$a+b \leftrightarrow a^{\prime}+b^{\prime}+c^{\prime}$.

Here $c^{\prime}$ may include many elements whose individual characteristics are irrelevant to the considered interaction.

The reader should not get the misleading impression from (1), that particle identity is preserved during transformation. In fact this is only true for particle excitation with rotational, vibrational and electronic internal changes. Here the purpose of using the correspondence $b \rightarrow b^{\prime}$ is to remind us that the source of $b^{\prime}$ particles was the target $b$. We will present two examples next, one related to high energy interactions and the other concerned with low energy chemical reactions.

As a first example consider the incidence of solar energy on a molecular oxygen gas. Then $a$ is identified as the incident solar radiation, $b$ as a molecular oxygen particle and the process as dissociative. Thus $b^{\prime}$ will be two atomic oxygen particles and $a^{\prime}$ will be a reduced energy radiation. No additional products will appear.

As a second example consider the reaction:

$e+\mathrm{NO}^{+} \rightarrow \mathrm{N}+\mathrm{O}$.

Here we easily identify the electron as the incident element $a, \mathrm{NO}^{+}$as the target particle $b$. One also considers $b^{\prime}$ as the atomic nitrogen and $c^{\prime}$ as the atomic oxygen, based on the knowledge that this reaction belongs to a set of nitrogen chemistry reactions of the upper atmosphere (Torr and Torr, 1979). As a rule of thumb, for these low-energy inelastic collisions, the higher temperature reactant is usually the incident element $a$ and the lower temperature one is the target particle $b$.

In classical mechanics Eq. (1) is governed by the equations of conservation of matter, momentum and energy. The first determines the characteristics of $c^{\prime}$. The last two are written as:

$p_{a}+p_{b}=p_{a}^{\prime}+p_{b}^{\prime}+p_{c}^{\prime}$,

$w_{a}+w_{b}=w_{a}^{\prime}+w_{b}^{\prime}+w_{c}^{\prime}$,

where $p$ denotes the momentum and $w$ the energy. Subscripts are attached to distinguish the element to which the symbols refer. Care must be exercised to properly use Eq. (3) since implicit to any sort of transformation is an unmanifested energy variation. Some authors (e.g., Burgers, 1969; Eccles and Raitt, 1992) prefer to include this energy in Eq. (3). If $a$ has eventually a radiative form one must use de Broglie's hypothesis with:

$p_{a}=2 \pi \hbar / \lambda_{a}$,

$w_{a}=2 \pi \hbar / v_{a}$

where $\lambda_{a}$ and $v_{a}$ are respectively the wavelength and wave frequency of the radiation and $\hbar$ is Planck's constant.

An alternative and sometimes preferred form in which to express Eqs. (2) and (3) is by means of the Hamiltonian of the system, $H$, which is a function of $N$ generalized coordinates, $q_{i}$, and $N$ corresponding momenta, $p_{i}$. Here, $N$ is the degrees of freedom of the system. The Hamiltonian is the total energy of the system $E$, consisting of a kinetic part $T$, and potential part, $U$. The conservation equations become:

$\sum_{i} \partial H / \partial p_{i}=\sum_{j} \partial H^{\prime} / \partial p_{j}$

$H=E$.

In 1926 Erwin Schrodinger put forth a theory which conforms the wave properties of de Broglie's with the conservation equations of classical mechanics. He assumed that Eq. (6) holds in the form:

$H \psi=E \psi$.

Here, now, the total energy stands as the eigenvalue of the second order differential operator $H$ and $\psi$ is a complex function of the coordinates and time.

For shortness we will not comment on the interesting resources provided by these semiclassical mechanics but refer the reader to Keller (1985 and references therein). Instead we show how its application to our problem help us to get a better insight into the semiclassical mechanics usefulness.

To start with, inspection of Eq. (1) allows us to characterize our problem as that of a binary interaction with, eventually, a multi-product output. Its similarity with that of elastic collisions is essential to the understanding of the proposed approach to its solution.

The Hamiltonian formulation together with the elastic collision analogy (see Liboff, 1969; Zamlutti, 1994) makes it possible to establish a clear distinction between the interacting elements, consider $a$ as the active incident, kinetic energy carrier element and particle $b$ as the passive target, potential energy holder. It is then possible to identify, by convenient rearrangement of the terms in Eqs. (2) and (3), the total amount of momentum (or energy) lost by $a$ as the sum of the momentum (or energy) gain of the target and other products. Therefore, from the classical mechanics viewpoint a solution to this problem can be determined.

As long as we are dealing with a stationary force field for our problem, the $\psi$ function can be put in the 
well-known form:

$\psi=A \exp \left[\frac{i}{\hbar}(p x-E t)\right]$

which holds for all wave propagating phenomena that obey de Broglie's conditions in Eq. (4). Here $x$ stands for the interparticle distance and the quantum mechanics formulation is valid for particles collision. To use it for radiative incident elements one can apply Eq. (4) to get an equivalent electron-particle interaction problem. The equivalent problem is obtained for an incident electron with velocity $v^{*}$ given by:

$v^{*}=p_{a} / m_{e}$

where $m_{e}$ is the incident electron mass.

The differential form of the Hamiltonian operator can be easily determined from Eq. (8) to be of the form:

$H=-\left(\hbar^{2} / 2 m_{a}\right) \nabla^{2}+U$,

where $m_{a}$ is the incident particle mass (see Savelyev, 1981).

The quantum mechanics solution to our problem is the most convenient one (see Lane, 1980; Rudd et al., 1992; Massey and Burhop, 1969). However, its complexity prevents its use when obtaining simple analytical expressions of easy implementation in upper atmosphere modeling. For this case we suggest the classical mechanics approach, with the caution of using the total cross section obtained from quantum mechanics methods when higher accuracy is necessary.

No matter what the chosen approach, the main problem to get a solution is the appropriate representation of the potential energy $U$. In the present case, because of the strong interaction between the colliding particles no simplified form can be used for the potential, which must account not only for the long-range effect but also for the close-range influence of the inner shell structure as well as for the incident element. It is written as:

$U=U_{s}+U_{e x}+U_{p}$

where $U_{s}$ is the electrostatic potential due to the unperturbed charge distribution within the target shell, $U_{e x}$ is the effective energy received from the incident element and $U_{p}$ is the polarization potential felt by the incoming particle at long distances (see Bhattacharyya and Goswami, 1983; Morrison, 1983; Jain et al., 1983).

The reader familiar to elastic collisions is also accustomed to use the long-range potential, $U_{p}$, which is usually of the form $k / x^{\prime \prime}$. Here to make the long-range effects conform with the short-range ones Bhattacharyya and Goswami (1983) recommend the use of:

$U_{p}=\left(k / x^{\prime \prime}\right)\left[1-\exp -\left(x / x_{c}\right)^{6}\right]$,

where $x_{c}$ is the cutoff distance usually assumed to be twice the Bohr radius.

Within the cutoff distance the incident element feels a Coulomb potential given by:

$U_{s}=\left(-\sum_{i=1}^{M} \frac{Z_{i}}{\left|x-X_{i}\right|}+\sum_{j=1}^{N_{j}} \frac{1}{\left|x-x_{j}\right|}\right) q e$, where $Z_{i}$ is the number of protons in the $i$ nucleus, $q$ is the charge of the incident particle, $e$ is the magnitude of the electron charge, $x_{j}$ are the coordinates of $N_{j}$ bound electrons and $x_{j}$ of $\mathrm{M}$ nuclei. All these coordinates are referred to the center of mass of the target particle.

The exchange potential, $U_{e x}$, is so called to emphasize that it effectively accounts for the energy interchange between the incident element and the target during the encounter. The energy is transferred in discrete amounts sufficient to drive electronic excitation (atomic or molecular target), vibrational and rotational excitation (molecular target). Larger amounts of energy may even produce ionization (atomic or molecular target) or dissociation (molecular targets). It can be written in the form:

$$
\begin{aligned}
U_{e x}= & \left(U_{i}^{2} / T\right) H^{*}\left(T-U_{i}\right)+\left(U_{d}^{2} / T\right) H^{*}\left(T-U_{d}\right) \\
& +\left(U_{e}^{2} / T\right) H^{*}\left(T-U_{e}\right) \\
& +T^{-1}\left\{\hbar \omega_{v} I\left[\left(T-U_{v}\right) /\left(\hbar \omega_{v}\right)\right]\right\}^{2} H^{*}\left(T-U_{v}\right) \\
& +T^{-1}\left\{\hbar \omega_{1} I\left[\left(T-U_{r}\right) /\left(\hbar \omega_{1}\right)\right]\right\}^{2} H^{*}\left(T-U_{r}\right),
\end{aligned}
$$

where $U_{i}$ and $U_{d}$ are respectively the ionization and dissociation energies, $U_{e}$ is the energy required for an electron to jump from one low level of energy to the other higher energy level within the electronic configuration of the target particle. $U_{v}$ and $U_{r}$ are respectively the vibrational and rotational energies and $\omega_{v}$ and $\omega_{1}$ are respectively the vibrational and rotational angular frequencies. $I(y)$ denotes the largest integer of the real value $y$ and $H^{*}(y)$ is the Heaviside unit function with the value 1 for $y>0$ and 0 for $y<0$.

Unfortunately, not all the required elements for Eq. (14) are available for use. We must emphasize the importance of the very useful compilations done by Itikawa et al. (1986; 1989), who collected together the necessary values for $\mathrm{N}_{2}$ and $\mathrm{O}_{2}$. The theoretical derivations to determine the energy levels is based on the conservation of the internal equilibrium for atoms and molecules, expressed by means of the Schrodinger equation (see, for instance, Takayanagi and Itikawa, 1970; Savelyev, 1981; Thompson, 1983).

The reader can now understand why Eq. (3) had to be handled with caution. The exchange potential holds a special place since it is purely a quantum mechanical entity. Its vibrational, rotational and electronic internal energies have been used by Morse (1964) and McCormack (1968) for the purposes of including the variations of internal energy of the particles in thermodynamics and fluid theory respectively. Using classical mechanics to account for $U_{i}$ and $U_{d}$ in chemical reactions Eccles and Raitt (1992) included the respective energy variations explicitly in Eq. (3). Here the use of $U_{e x}$ makes this inclusion unnecessary.

\section{Aspects of the reactive dynamics}

All transformations occurring in upper atmosphere particles are produced by internal energy variations (due to absorption or loss processes) and involves essentially 
three aspects:

1. The statistical rules of the transformation

2. Computation of the features after the transformation

3. The geometrical characteristics of the transformation

These aspects present strict similitude with those of a binary collision and this led Burgers (1969) to propose the binary kinetic collision term of BGK-type to model the chemistry and resulting dynamics. Since one particle specie is transformed into another specie a chemical reaction is understood to have occurred. This is probably the reason for the inclusion of the word reactive and the term is called a reactive collision due to particle transformation. Eccles and Raitt (1992) started their modeling using a chemical reaction for production and another for the loss of the considered particle. Here we adopt a more formal procedure and describe all sorts of transformations as binary interactions of the form (1). The two approaches are equivalent and thus a chosen specie $s$ is produced if $b^{\prime} \equiv s$ and is lost if $b \equiv s$ in (1). Stated this way and provided that the conservation Eqs. (2) and (3) holds we can moreover assume that the reverse process of (1) is possible.

The characterization of the present problem as a binary interaction constitutes an alternative approach to circumvent the actual many body problem of particle transformations (see, for instance, Takayanagi and Itikawa, 1970; Lane, 1980; Morrison, 1983; Thompson, 1983).

Of course, the obtained results are expected to be, at most, satisfactory approximations of the actual solution (see Keller, 1985).

The binary interaction approach follows the major steps outlined in Zamlutti (1994). We then consider that the independent statistical aspects of the incident element and the target particle are characterized respectively by uncorrelated distribution functions $f_{a}$ and $f_{b}$. The statistical characteristics during the encounter are described by a joint distribution function, $f_{t}$, such that at a given instant $\mathrm{t}, f_{t} d \underline{v}_{b} d \underline{r}_{b}$ expresses the number of particles, with velocities between $\underline{v}_{b}$ and $\underline{v}_{b}+d \underline{v}_{b}$ and positions between $\underline{r}_{b}$ and $\underline{r}_{b}+d \underline{r}_{b}$, which are transformed under the action of $a$. Here underlined symbols denote three-dimensional vector entities of the phase space. Thus, $d \underline{v}_{b}$ represents a velocity space volume element and $d \underline{r}_{b}$ the coordinate space volume element. For the considered uncorrelated behaviors of $a$ and $b$ it can be expressed as:

$f_{t}=f_{a} f_{b}$.

The perturbation produced in the distribution function of the $b$ particles due to each transformed particle is:

$\Delta f_{t}=f_{t}^{\prime}-f_{t}$.

The total modification in the distribution of the $b$ particle resulting from all transformed particles, during the time internal $d t$, is given by

$\left(\Delta f_{t}\right)_{b}=\iint \Delta f_{t} d \underline{v}_{b} d \underline{r}_{b}$,

where each integral sign with nonspecified limits represents one volume integration over the entire space. The reader familiar to elastic collisions theory is accustomed to consider Eq. (17) as the amount of disturbance produced in the distribution function of the incident element $a$ by encounters with the totality of targets $b$. Conversely, here, by the reciprocity principle the same amount represents the number of targets $b$ converted into $s$ particles by the actions of $a$. If, for instance, $a$ is a spectral radiation line Eq. (17) will measure the number density of produced particles $s$.

The time rate of transformation is then:

$\left(\delta f_{t}\right)_{b}=\left(\Delta f_{t}\right)_{b} / d t$

So far Eq. (18) refers to a particular set of characteristics $\left(p_{a}, w_{a}\right)$ of the incident element $a$. To account for the entire range of energy variation one must integrate Eq. (18) over this range. To make it general enough and appropriate to transport equations of the $s$ particles, we write the integral as:

$(\delta Q)_{p}=+\int Q\left(\underline{v}_{s}\right)\left(\delta f_{t}\right)_{b} d w_{a}$,

$(\delta Q)_{l}=-\int Q\left(\underline{v}_{s}\right)\left(\delta f_{t}\right)_{b} d w_{a}$,

where $Q\left(\underline{v}_{s}\right)$ are the velocity moments of the considered specie. Subscripts $p$ and $l$ denote produced and lost respectively. The negative sign indicates that the loss term for the $s$ particle is actually computed using the expression for the production of the particles derived from it. The analogy of Eq. $(19 a, b)$ with the velocity moments of the binary elastic collisions is evident. The earlier versions of Eq. (19a, b) derived by Burgers (1969) and Eccles and Raitt (1992) consider the integration over the velocity range of the incident particle, thus restricting their validity to particleparticle collisions. The present approach is general enough to account for all sorts of transformations. Its applications require some care since it must be remembered that:

1. We are considering moments of the actual chosen species $s$ in Eq. $(19 \mathrm{a}, \mathrm{b})$.

2. The integration is carried out over the incident energy range and therefore $f_{a}$ of Eq. (15) and subsequent expression must be expressed in energy terms.

To carry out the space integration of Eq. (17) the volume element $d \underline{r}_{b}$ is usually written as:

$d \underline{r}_{b}=S d l$,

with

$S=\int_{0}^{2 \pi} \int_{0}^{\pi} \sigma \sin \beta d \beta d \psi$

where $S$ is the total cross section of the target particle and $d l$ is the elementary distance along the path. Here $\sigma$ is the differential scattering cross section, $\beta$ the scattering angle and $\psi$ the angular variation perpendicular to the plane of incidence. The computation of $S$ has been the subject of extensive research (see Gallagher et al., 1988; Jain and Bauja, 1992; Rudd et al., 1992 and references therein). In spite of all the effort so far there still is no accurate simple expression of easy implementation in upper atmosphere modeling. For the purpose of this work then, to provide 
the reader with a simple method for introducing the effect of the reactive interactions we may use an approach which resembles the accepted Bethe-Born approximation (e.g., Heddle and Gallagher, 1989; Morrison, 1983; Rudd et al., 1992).

To compute the coordinates volume integral necessary for the case of particle transformations, we restrict the considerations first to short-range interactions. Thus, one can discard the polarization potential in Eq. (11). We are then left with a discrete loss of the incident energy due to the exchange potential and an interactive Coulomb interaction, due to the electrostatic potential. This situation is analogous to that of the elastic encounter of charged particles with its pertinent beam-beam geometry (Trajmar et al., 1983; Zamlutti, 1994), provided that we represent radiations-particle problems by the equivalent electronparticle models (see Sect. 2). Under these circumstances we are allowed to use the elastic collisions transport cross sections, $S_{1}$ and $S_{2}$, (see Schunk, 1977; Zamlutti, 1994) which account for the geometry of the interaction.

$$
\begin{aligned}
& S_{1}= \frac{\pi x_{c}^{2} U_{s}^{2}}{2\left(T-U_{e x}\right)^{2}} \ln \left[1+\frac{4\left(T-U_{e x}\right)^{2}}{U_{s}^{2}}\right], \\
& S_{2}= \frac{\pi x_{c}^{2} U_{s}^{2}}{\left(T-U_{e x}\right)^{2}}\left\{\left[1+\frac{4\left(T-U_{e x}\right)^{2}}{U_{s}^{2}}\right]-1\right. \\
&\left.+\ln \left[1+\frac{4\left(T-U_{e x}\right)^{2}}{U_{s}^{2}}\right]-1\right\}, \\
& S=2 \pi v_{r}^{-1}(2 k / \mu)^{1 / 2}=2 \pi x^{2}\left[U_{p} /\left(T-U_{e x}\right)\right]^{1 / 2},
\end{aligned}
$$

where $v_{r}=d l / d t$ is the magnitude of the relative velocity of the collision (see Zamlutti, 1994). The constant $k=$ $10^{-24} \mathrm{e}^{2} \mathrm{~g}(\mathrm{~cm} / \mathrm{sec})^{2} \mathrm{~cm}^{4}$ is that used for Maxwell molecule interaction (Banks, 1966) and $\mu$ is the reduced mass. These expressions are very similar to those determined for the Bethe-Born approximation (see Inokuti, 1971; Inokuti et al., 1978). The static potential is computed at the cutoff distance $x_{c}$

The use of Eqs. (15)-(18) and Eq. (20a, b) in Eq. (19a, b) (see Chapman and Cowling, 1970, for derivation and Zamlutti, 1994, for a review) gives:

$$
\begin{aligned}
& (\delta Q)_{p}^{*}=+\iint f_{a} f_{b} v_{r} \hat{Q}\left(\underline{v}_{b}\right) d w_{a} d \underline{v}_{b}, \\
& (\delta Q)_{l}^{*}=+\iint f_{t} f_{s} v_{r} \hat{Q}\left(\underline{v}_{s}\right) d w_{t} d \underline{v}_{s},
\end{aligned}
$$

where $\hat{Q}\left(\underline{v}_{b}\right)$ is the total integrated cross section for moment transference from $a$ to $b$. The subscript $t$ was used in Eq. (22b) to remind the reader that, in spite of the fact that production and loss equations are formally identical the participating reactants are different. The value of $\hat{Q}\left(\underline{v}_{b}\right)$ is as follows:

$$
\begin{aligned}
& \text { for } Q\left(\underline{v}_{b}\right)=m_{b}, \quad \hat{Q}=0 ; \\
& \text { for } Q\left(\underline{v}_{b}\right)=m_{b} \underline{v}_{b}, \quad \hat{Q}=\mu \underline{v}_{r} S_{1} ; \\
& \text { for } Q\left(\underline{v}_{b}\right)=m_{b} \underline{v}_{b}^{2} / 2, \\
& \hat{Q}=\left[\mu \underline{v}_{b} \cdot \underline{v}_{r}+\left(\mu^{2} / m_{b}\right) v_{r}^{2}\right] S_{1} ;
\end{aligned}
$$

$$
\begin{aligned}
& \text { for } Q\left(\underline{v}_{b}\right)=m_{b} \underline{v}_{b} \underline{v}_{b}, \\
& \begin{aligned}
\hat{Q}=\left[\mu\left(\underline{v}_{b} \underline{v}_{r}+\underline{v}_{r} \underline{v}_{b}\right)+2\left(\mu^{2} / m_{b}\right)\left(\underline{v}_{r} \underline{v}_{r}\right)\right] S_{1} \\
+\left(\mu^{2} / m_{b}\right)\left[v_{r}^{2} I-3 \underline{v}_{r} \underline{v}_{r}\right]\left(S_{2} / 2\right) ;
\end{aligned}
\end{aligned}
$$

for $Q\left(\underline{v}_{b}\right)=m_{b} v_{b}^{2} \underline{v}_{b} / 2$.

$$
\begin{aligned}
\hat{Q}=\{ & \mu\left[\left(\underline{v}_{b} \cdot \underline{v}_{r}\right) \underline{v}_{b}+\left(v_{b}^{2} / 2\right) \underline{v}_{r}\right]+\left(\mu^{2} / m_{b}\right)\left[\underline{v}_{r}^{2} \underline{v}_{b}\right] \\
& \left.+2\left(\mu^{3} / m_{b}^{2}\right) v_{r}^{2} \underline{v}_{r}\right\} S_{1}+\left\{( \mu ^ { 2 } / m _ { b } ) \left[\left(v_{r}^{2} / 2\right)\left(\underline{v}_{b} \cdot \mathbf{I}\right)\right.\right. \\
& \left.\left.-(3 / 2)\left(\underline{v}_{b} \cdot \underline{v}_{r}\right) \underline{v}_{r}\right]-\left(\mu^{3} / m_{b}^{2}\right) v_{r}^{2} \underline{v}_{r}\right\} ;
\end{aligned}
$$

with $\underline{v}_{r}=\underline{v}_{a}-\underline{v}_{b}$ (see Zamlutti, 1994). Here we considered the equivalent electron-particle model to simulate the effects of an incident radiation.

Thus far, the considerations were restricted to the required moment transference from $a$ to $b$ to transform them into $s$ particles. However, there are moments carried along from $b$ to $s$ during the transformation, since the $s$ particles are, in fact, modified versions of the $b$ particles. To compute this parcel one assumes that the totality of the $b$ particles undergoing the changes are transmuted into $s$ particles. Under these circumstances $f_{t}^{\prime}=0$ in Eq. (16). Then using Eqs. (15)-(18) and Eq. (20) in Eq. (19a, b) we obtain:

$$
\begin{aligned}
& (\delta Q)_{p}^{* *}=+\iint f_{a} f_{b} v_{r} S Q\left(\underline{v}_{s}\right) d w_{a} d \underline{v}_{b}, \\
& (\delta Q)_{l}^{* *}=-\iint f_{t} f_{s} v_{r} S Q\left(\underline{v}_{s}\right) d w_{t} d \underline{v}_{s},
\end{aligned}
$$

which accounts for the long-range moment variation of the $b$ particle due to the interaction.

The total moment variations amount to:

$$
\begin{aligned}
& (\delta Q)_{p}=(\delta Q)_{p}^{*}+(\delta Q)_{p}^{* *} \\
& (\delta Q)_{l}=(\delta Q)_{l}^{*}+(\delta Q)_{l}^{* *} .
\end{aligned}
$$

The computation of the RHS terms of Eq. (25a,b) depends on the particularities of the considered phenomenon. Thus, for solar radiation we may impose conditions that $m_{a}\left\langle\left\langle m_{b}, p_{a}\right\rangle\right\rangle p_{b}, w_{a}=c p_{a}$, where $c$ is the speed of light. Then:

$$
\begin{aligned}
& \text { a. for } Q\left(\underline{v}_{s}\right)=m_{s}, \quad(\delta Q)_{p}^{*}=0, \quad(\delta Q)_{l}^{*}=0, \\
& (\delta Q)_{p}^{* *}=2 \pi \chi_{c}^{2} m_{s} n_{b} c U_{p}^{1 / 2} \int w_{a}^{1 / 2} f_{a} d w_{a}, \\
& (\delta Q)_{l}^{* *}=-2 \pi(2 k / \mu)^{1 / 2} m_{s} n_{s} n_{t} \\
& \text { b. for } Q\left(\underline{v}_{s}\right)=m_{s} \underline{v}_{s}, \\
& (\delta Q)_{p}^{*}=\left(n_{b} \int w_{a} S_{1} f_{a} d w_{a}\right) \underline{e}_{\chi} \\
& (\delta Q)_{l}^{*}=-\mu n_{t} n_{s}\left\langle v_{r}\right\rangle S_{1}\left(\underline{u}_{t}-\underline{u}_{s}\right) \\
& (\delta Q)_{p}^{* *}=0, \quad(\delta Q)_{l}^{* *}=0 \\
& \text { c. for } Q\left(\underline{v}_{s}\right)=m_{s} \underline{v}_{s}^{2}, \\
& (\delta Q)_{p}^{*}=n_{b}\left(m_{b} c^{2}\right)^{-1} \int w_{a}^{2} S_{1} f_{a} d w_{a} \\
& (\delta Q)_{l}^{*}=n_{t} n_{s}\left\langle v_{r}\right\rangle S_{1}\left[\left(-\mu^{2} / m_{t} m_{s}\right) 3 K T_{t}\right. \\
& \left.\quad-\left(\mu / m_{s}\right)\left(\mu-m_{s}\right) m_{s}^{-1} 3 K T_{s}\right] \\
& (\delta Q)_{p}^{* *}=\pi x_{t}^{2}\left(3 K T_{s}\right) n_{b} c U_{p}^{1 / 2} \int w_{a}^{-1 / 2} f_{a} d w_{a} \\
& (\delta Q)_{l}^{* *}=-\pi(2 k / \mu)^{1 / 2}\left(3 K T_{s}\right) n_{t} n_{s}
\end{aligned}
$$


with $w_{a}=T-U_{e x}$ : Here $K$ is Boltzmann's constant and $T_{s}$ the temperature of the $s$ particles. It is also understood that the $b$ particles have no flow velocity and $f_{a}(\lambda, v, t)$ is the distribution function of the incident photons. The other symbols denote the unit vector of the direction of incidence, $\underline{e}_{\chi}$, the flow velocity, $\underline{u}$, the ensemble average, $\langle\cdot\rangle$. To compute $(\delta Q)_{l}^{*}$ we considered $s$ as the target particle (i.e., $T_{t}>T_{s}$ ). If the converse holds subscripts $t$ and $s$ must be interchanged.

To be consistent with upper atmosphere physics we preserved the radiation-particle expressions only for the production terms, since the loss of the $s$ particles is, in fact, due to inelastic particle-particle encounters. These expressions are valid no matter the amplitude of the incident energy. Therefore they account not only for the effects on the translational temperature of internal energy variation of the interacting particle (Morse, 1964; McCormack, 1968) but also for the effects of all chemical reactions occurring in the upper atmosphere (Burgers, 1969; Eccles and Raitt, 1992).

Burgers (1969) and Eccles and Raitt (1992), using a particle-particle collision model, preferred not to split the computation of Eq. (19a,b) as we did in the present approach. Instead, Eccles and Raitt (1992), considered that the energy requirement of Eq. (1) was used to increase the thermal velocity of the $s$ particles. This assumption may not be quite valid, since during the innermost encounter rotational and vibrational excitation of the target particle may occur. Thus, the knowledge of the underlying inner shell structural changes of the target is essential, to the proper interpretation of the distribution of the incident energy lost among the resulting products. Inokuti (1971) computed the target particle cross section offered to the incident element and obtained results in agreement with Eq. (21a). Since he put his emphasis on the incident element his cross section only accounts for the contribution of Eq. (22a) to the study of particles transformation.

To complete our presentation we must show the kinetic form that the production-loss term assumes. With our considerations for short- and long-range components after substitution of (15)-(17) and $(20 \mathrm{a}, \mathrm{b})$ in (18) we obtain:

$\left(\delta f_{t}\right)_{p}=\left(S_{1}+S\right) f_{a} \int v_{r} f_{b} d \underline{v}_{b}$,

$\left(\delta f_{t}\right)_{l}=-\left(S_{1}+S\right) f_{t} \int v_{r} f_{b} d \underline{v}_{b}$,

where use was also made of the transport collision cross sections for Coulomb interactions (short-range) and Maxwell molecules interactions (long-range) given by Zamlutti (1994). The integral in Eq. (26a) is identified as the average relative velocity of the interacting elements, approximated by:

$\left\langle v_{r}\right\rangle=\left(\frac{8}{3} \pi\right)^{1 / 2}\left[\left(w_{a} / p_{a}\right)^{2}+3 K T_{b} / m_{b}\right]^{1 / 2}$,

see Zamlutti (1994).

Finally, the proposed approach allows us to evaluate the scattering angle $\beta$ by:

$\beta=\pi-2 \theta$,

with $\tan \theta=2 w_{a} / U_{p}$.
As in the BGK approach for elastic collisions Eq. $(26 \mathrm{a}, \mathrm{b})$ gives only an approximation to model the actual microscopic behavior of the transformation processes.

\section{The theoretical problems of the reactive dynamics}

It was mentioned that particles transformations occur through an innermost interaction between the incident element and the target particle. They promote changes in the internal equilibrium of the considered particle being then a many body phenomenon. To model it properly quantum mechanics formalisms are considered in the chemical literature (e.g., Burke, 1979; Lane, 1980; Morrison, 1983; Thompson, 1983).

The Hamiltonian of the system is written as:

$H=H_{m}+T+U_{s}$,

where $H_{m}$ is the Hamiltonian of the isolated molecule, $T$ the kinetic energy operator for the scattering element and $U_{s}$ is the electrostatic interaction potential between the incident element and the molecule. Here, we circumvent the difficulties of the quantum mechanics approach using relation (7) to replace all the differential operators by equivalent kinetic and potential energies. Then the quantum mechanics problem is reduced to a classical mechanics one. When this is done the quality of the results obtained is also reduced. However, as pointed out by Inokuti (1971) because of the peculiar nature of the Coulomb force the classical mechanics cross section retains its form in quantum mechanics. We believe that as far as simple methods are concerned we have done our best in the present work.

Because of its flexibility, the proposed approach has a wide range of applications which comprise most of the upper atmosphere needs regarding particles transformations. It is more appropriate to the cases in which the target particle structure is preserved. However, it can also be adapted to study association and dissociation of particles, it can be adapted to study also particle association and dissociation. However, it depends on knowledge of the underlying chemical processes to provide an adequate representation of the considered phenomenon.

The presented matter was based on a theory that considered the motion of the colliding particles confined to a plane (particle-particle encounters) or equivalently to plane wave geometry (radiation-particle interactions). It is comparable to the plane wave Born approximation (PWBA) used in quantum mechanics. The shortcomings of its validity are discussed in Rudd et al. (1992) and refer to the behavior of the scattered element, interacting with the ejected electron during ionizing proton-particle collisions. This difficulty deals essentially with the modification undergone by the interacting potentials that affect the scattering element during the encounter. One can handle this trouble by considering the outer shell scattered path as a second interaction problem with its pertinent geometry. This type of solution has also been used in the quantum mechanics approaches to deal with the electronparticle collision problem (see Burke et al., 1987). 
Another criticism of the validity of the cross sections of Inokuti's type is that they do not agree with experimental data at low incident energies. As pointed out in this work the contribution of $S_{1}$ and $S_{2}$ constitutes only one part of the total cross section. Thus, as far as this limitation is concerned, the present approach seems to be in the right direction.

The aforementioned strategy of using an exchange potential to account for energy exchanges between the interacting elements poses another difficulty to the implementation of the method proposed in this work. To overcome this hindrance there are compilations like those of Itkawa et al. $(1986,1989)$ to which one can resort to help in determining the necessary parameters. The computation of the characteristic energies and angular frequencies of the target can also be derived (e.g., Savelyev, 1981; Schneider et al., 1979; Thompson, 1983) using theoretical approaches. Other alternative ways to treat this matter are mentioned in Morrison (1983).

Care must be exercised to use the presented theory properly. As a guideline we recall that:

a. Solar radiation and intermediate energy particle precipitation drives particle transformations where the term $(\delta Q)_{p}^{*}$ is very important (particle dissociation or ionization).

b. Low energy processes drive the formation of excited and metastable species (electronic, vibrational and rotational excitations).

c. Very low energy processes include most of the subsequent chemical reactions that occur in the upper atmosphere with reduced importance of $(\delta Q)_{p, l}^{*}$.

d. Associative and recombination processes are reactions where $a$ and $b$ combine to produce $b^{\prime} \equiv a b$ and release a radiative energy ( $c^{\prime}$ product) in Eq. (1).

e. During high incident energies carried by electrons there is a chance that their velocity will be large enough so that no particle transformation occurs. Then $U_{e x} \rightarrow 0$ and $(\delta Q)_{p}^{* *}=0$. The target just "sees" the passing electron and receives from it an impulsive moment given by $(\delta Q)_{p}^{*}$ (see Lane, 1980).

In this reminder it is understood that:

I. $T<10 \mathrm{eV}-$ very low energies.

II. $10 \mathrm{eV}<T<100 \mathrm{eV}$ - low energies

III. $100 \mathrm{eV}<T<1000 \mathrm{eV}$ - intermediate energies

IV. $1000 \mathrm{eV}<-$ high energies

In addition it seems clear that unless the incident element interacts for some time with the target there is no chance for a transformation to occur. Therefore the most complex form of transformations hold for every low incident energies (see Massey and Burhop, 1969; Lane, 1980; Morrison, 1983). In order to give an indication of this problem we found the characteristic times:

$10_{s}^{-12}>$ rotational excitation time

$10_{s}^{-13}$ to $10_{s}^{-8}-$ dissociation time

$10_{s}^{-14}>$ vibrational excitation time

$10_{s}^{-17}>$ ionization time.

(see Hays et al., 1978; Morrison, 1983; Galagher et al., 1988) and computed the following transit time for an electron within the inner shell:

$t=1.6 \times 10_{s}^{-15}-$ for $10 \mathrm{eV}$

$t=5.4 \times 10_{s}^{-16}-$ for $100 \mathrm{eV}$

$t=1.6 \times 10^{-16}-$ for $1000 \mathrm{eV}$.
Thus, the only process that may occur above $10 \mathrm{eV}$ is ionization.

The physical reasoning about the transit time is the factor responsible for our particular choice of the form of the exchange energy in Eq. (14). In it, a damping factor of the form $a /(a+y)$, for growing $y$, was introduced to account for the "closure" of each "excitation channel".

\section{Discussion}

In so far as the methodology was concerned in the preceding material we introduced the grounds on which our method was based, detailed its mathematical expressions and commented on the limitations to its validity pointed out in the current literature. The wide range of chemical processes to which the proposed approach is applicable prevents the presentation of a satisfactory set of examples that could give the reader a fair idea of the full extent of its use. Nevertheless, the presented case of solar radiation effects allows us to give the reader some insight into the usefulness of the suggested procedure.

The reader familiar to upper atmosphere studies recognizes that for $Q\left(\underline{v}_{s}\right)=m_{b^{\prime}},(\delta Q)_{p}^{* *}$ is the rate of production of the $s$ particles where we can identify:

$k=2 \pi x_{c}^{2} c-$ reaction rate

$\eta(\lambda)=\left(U_{p} / w_{a}\right)^{1 / 2}-$ photoabsorption efficiency

$n_{a} n_{b}=n_{b} \int f_{a} d w_{a}-$ product of reactant densities

where $n_{a}$ is the density of photons (e.g., Schunk, 1983; Hays et al., 1978). Also for $Q\left(\underline{v}_{s}\right)=m_{b} v_{b}^{2},(\delta Q)^{*}$ is the rate of energy transfer from the solar flux to the ambient gas where by inspection one sees that:

$d \bar{E} / d x=n_{b} w_{a} S_{1}-$ rate of energy transfer

$\phi(\lambda)=c f_{a}-$ solar flux

$P_{a} /\left(m_{b} c\right)$ - mass reduction rate

where the LHS is the usual form found in the literature for energy transfer from flow type processes (e.g., Schunk, 1983; Dalgarno, 1969). This shows the consistency of our approach with the upper atmosphere techniques as far as the correspondence of expressions is concerned.

A next step in comparison is to show the consistency of our method with recent works on the same field. To do this we chose the work of Jain and Baluja (1992). One first observes that these authors split the potential energy of Eq. (11) into a real and an imaginary parts. Their imaginary part corresponds to our exchange potential $U_{e x}$ and accounts for the effect of the actual energy absorbed by the target. The equivalent cross section should agree with Eq. (21a). In fact we computed $U_{s}$ according to the assumption that at the cutoff distance it must be equal to the quadrupole moment potential (e.g., Bhattacharyya and Goswami, 1983) for $\mathrm{H}_{2}, \mathrm{~N}_{2}$ and $\mathrm{O}_{2}$. Thus one may write the approximation:

$U \cong-\left(Q / x_{c}^{3}\right) P_{2}(\cos \theta)\left[1-\exp -\left(x / x_{c}\right)^{6}\right]$

to be used in Eq. (21a). Here $Q$ is the quadrupole moment and $P_{2}(\cos \theta)$ is the Legendre polynomial. Since $S_{1}$ itself is already an integrated cross section one must consider the average value of the Legendre polynomial in Eq. (30). We considered the low incident electron energy of $20 \mathrm{eV}$ and 
$N_{2}$ molecules as target. The ionization potential is $15.6 \mathrm{eV}$ and $Q=-0.95 a_{0}^{2} e^{2}$. Computing $S_{1}$ we got the value $0.21 \times 10^{-16} \mathrm{~cm}^{2}$ whereas Jain and Baluja (1992) obtained $0.15 \times 10^{-16} \mathrm{~cm}^{2}$. The $35 \%$ discrepancy is irrelevant considering the simplicity of the employed methodology and the crude form of obtaining the necessary parameters.

This example is illustrative also of the fact that the proposed form, similar to that of Inokuti (1971), properly satisfies our purpose in the present work and did not show any drastic failure at low incident energies. This improvement may be due to the procedure used in this work to handle the exchange potential during the interaction.

A final step in comparisons concerns the proper use of the semiclassical mechanics to conform the problem of quantum mechanics into classical mechanics formulations. Eccles and Raitt (1992) considered the problem of exchanged energy from a pure classical mechanics standpoint and neglected the cross-sectional difference between short- and long-range interactions. This may cause difficulties when the after-transformation potential interactions are different from the initial ones. This problem was commented on in the last section. Here we overcame this hindrance by properly splitting the short- and longrange in accordance with what is actually consistent with quantum mechanics formalisms. Another shortcoming of the Eccles and Raitt (1992) model is that is is not suitable to radiative incident energy. As a matter of fact these authors circumvent the problem by assuming a known photoionization production rate in their example. Here the use of de Broglie relations helped us to cope correctly with the predicaments of this sort of transformation in agreement with quantal models.

Finally, to summarize the outstanding aspects of the proposed method we call attention to:

1. It is a self-consistent procedure which requires the knowledge of the distribution functions and excitation parameters to adequately model the production and loss terms.

2. The theory conforms to more sophisticated results obtained by other authors.

3. There is a one-to-one formal correspondence of our results with traditionally accepted techniques used to predict the appearance of excited and metastable particles specie.

4. One can use our approach for problems like dissociation and association, ionization and recombination and exchange problems of upper atmosphere particles.

\section{Conclusions}

A thorough revision on the grounds necessary to develop a proper form for the production and loss terms of the Boltzmann equation was completed and a prospective model for them was presented. The proposed approach was shown to present satisfactory agreement with the current methodology on the matter. The resulting terms proved to be simple enough to allow for implementation in upper atmosphere modeling. Also the proposed model is self-consistent to the extent that all its necessary elements can be easily computed with the presented expressions. Only a few necessary parameters must be introduced from other works.

The theory presented is applicable to most of the phenomena involving particle transformations that occur in our environment, which range from low energy changes such as those of chemical reactions to high energy particle precipitation in the auroral upper atmosphere.

Acknowledgements. Support for this work came through Instituto Nacional de Pesquisas Espaciais, from FINEP under contract 537/CT. The author received also a complementary fellowship from Conselho Nacional de Desenvolvimento Cientifico e Tecnológico under the process 300901/90 (RN). The computer facility that helped the preparation of the manuscript was provided by Fundação de Amparo à Pesquisa do Estado de São Paulo.

The Editor in Chief thanks J. V. Eccles and another referee for their help in evaluating this paper.

\section{References}

Banks, P., Collision frequencies and energy transfer ions, Plant. Space Sci., 14, 1105, 1966.

Bates, D. R., The thermosphere, Proc. R. Soc. London, 236, 206, 1956.

Bhattacharyya, P. K., and K. K. Goswami, Elastic and rotational excitation of the oxygen molecule by intermediate-energy electrons, Phys. Rev. A., 28, 713, 1983.

Burgers, J. M., The construction of equations of motion for reacting gases, Presented at Meeting of the Soc. Eng. Sci., East Lansing, Michigan, USA, 1964.

Burgers, J. M., Flow equations for composite gases, Academic Press, New York, 1969.

Burke, P. G., Theory of low energy electron-molecule collisions, Adv. Atom. Molec. Phys., 15, 471, 1979.

Burke, P. G., C. J. Noble, and P. Scott, R-matrix theory of electron scattering at intermediate energies, Proc. R. Soc. London A, 410, 289, 1987.

Chapman, S., and T. G. Cowling, The mathematical theory of nonuniform gases, Cambridge University Press, London, 1970.

Dalgarno, A., Inelastic collisions at low energies, Can. J. Chem., 47, 1723, 1969.

Eccles, J. V., and W. J. Raitt, Reactive collision terms for fluid transport theory, Planet. Space Sci., 40, 47, 1992.

Gallagher, J. W., C. E. Brion, J. A. R. Samson, and P. W. Langhoff, Absolute cross sections for molecular photoabsorption, partial photoionization, and ionic photofragmentation processes, J. Phys. Chem. Ref. Data, 17, 9, 1988.

Hays, P. B., D. W. Rusch, R. G. Roble, and J. C. G. Walker, The $0 \mathrm{I}$ (6300 A) airglow, Rev. Geophys. Space Phys., 16, 225, 1978.

Heddle, D. W. O., and J. W. Gallagher, Measurements of electron impact optical excitation functions, Rev. Mod. Phys., 61, 221, 1989.

Inokuti, M., Inelastic collisions of fast charged particles with atoms and molecules - the Bethe theory revisited, Rev. Mod. Phys., 43, 297, 1971.

Inokuti, M., Y. Itikawa, and J. E. Turner, Addenda: inelastic collisions of fast charged particles with atoms and molecules - The Bethe theory revisited, Rev. Mod. Phys., 50, 23, 1978.

Itikawa, Y., M. Hayashi, A. Ichimura, K. Onda, K. Sakimoto, K. Takayanagi, M. Nakamura, H. Nishimura, and T. Takayanagi, Cross sections for collisions of electrons and photons with nitrogen molecules, J. Phys. Chem. Ref. Data, 15, 985, 1986.

Itikawa, Y., A. Ichimura, K. Onda, K. Sakimoto, K. Takayanagi, Y. Hatano, M. Hayashi, H. Nishimura, and S. Tsurubuchi, Cross sections for collisions of electrons and photons with oxygen molecules, J. Phys. Chem. Ref. Data, 18, 23, 1989. 
Jain, A., and K. L. Baluja, Total (elastic plus inelastic) cross sections for electron scattering from diatomic and polyatomic molecules at $10-5000 \mathrm{eV}: \mathrm{H}_{2}, \mathrm{Li}_{2}, \mathrm{HF}, \mathrm{CH}_{4}, \mathrm{~N}_{2}, \mathrm{CO}, \mathrm{C}_{2} \mathrm{H}_{2}, \mathrm{HCN}, \mathrm{O}_{2}, \mathrm{HCl}$, $\mathrm{H}_{2} \mathrm{~S}, \mathrm{PH}_{3}, \mathrm{SiH}_{4}$, and $\mathrm{CO}_{2}$, Phys. Rev. A., 45, $2202,1992$.

Jain, A., S. S. Tayal, L. C. G. Freitas, and L. M. Tao, Electron-linearpolyatomic-molecule elastic scattering at intermediate and high energies in a two-potential coherent approach: application to $\mathrm{C}_{2} \mathrm{H}_{2}$, J. Phys. B, 16, L99, 1983.

Keller, J. B., Semiclassical mechanics, Siam Rev., 27, 485, 1985.

Lane, N. F., The theory of electron-molecule collisions, Rev. Mod. Phys., 52, 29, 1980.

Liboff, R. L., Introduction to the theory of kinetic equations, John Wiley, New York, 1969.

Massey, H. S. W., and E. H. S. Burhop, Electronic and ionic impact phenomena, Oxford University Press, Oxford, UK 1, Chap. 8, 1969.

McCormack, F. J., Kinetic equations for polyatomic gases: the 17-moment approximation, Phys. Fluids, 11, 2533, 1968.

Morrison, M. A., The physics of low-energy electron-molecule collisions: a guide for the perplexed and the uninitiated, Aust. J. Phys., 36, 239, 1983.

Morse, T. F., Kinetic model for gases with internal degrees of freedom, Phys. Fluids, 7, 159, 1964.

Rudd, M. E., Y. K. Kim, D. H. Madison, and T. J. Gay, Electron production in proton collisions with atoms and molecules: energy distributions, Rev. Mod. Phys., 64, 441, 1992.
Savelyev, I. V., Physics a general course, vol III, Mir Publishers, Moscow, 1981

Schneider, B. I., M. LeDourneuf, and P. G. Burke, Theory of vibrational excitation and dissociative attachment: an R-matrix approach, J. Phys. B, 12, L365, 1979.

Schunk, R. W., Mathematical structure of transport equations for multispecies flows, Rev. Geophys. Space Phys., 15, 429, 1977.

Schunk, R. W., The terrestrial ionosphere, Solar Terr. Phys., 609, 1983, D. Reidel, Dordrecht

Takayanagi, K., and Y. Itikawa, The rotational excitation of molecules by slow electrons, Adv. At. Mol. Phys., 6, 105, 1970.

Thompson, D. G., The vibrational excitation of molecules by electron impact, Adv. At. Mol. Phys., 19, 309, 1983.

Torr, D. G., and M. R. Torr, Chemistry of the thermosphere and ionosphere, J. Atoms. Terr. Phys., 41, 797, 1979.

Torr, M. R., and D. G. Torr, The role of metastable species in the thermosphere, Rev. Geophys. Space Phys., 20, 91, 1982.

Trajmar, S., D. F. Register, and A. Chutjian, Electron scattering by molecules, II: experimental methods and data, Phys. Rep., 97, 219, 1983.

Zamluti, C. J., Transport equations for multicomponent isotropic and anisotropic high speed space plasmas, Planet. Space Sci., 42, 557, 1994. 